\title{
Probable Person-to-Person Transmission of Legionnaires' Disease
}

TO THE EDITOR: Legionnaires' disease is an often severe form of pneumonia that is typically acquired by susceptible persons (e.g., elderly persons and smokers) through inhalation of aerosols that contain legionella species. ${ }^{1-4} \mathrm{~A}$ cluster of cases of this disease occurred in Vila Franca de Xira, Portugal, in 2014. ${ }^{5}$

One of the first cases of disease in this cluster occurred in a 48-year-old man (Patient 1), a smoker, who had been employed since October 6,2014 , as a maintenance worker at an industrial cooling tower complex in Vila Franca de Xira that was subsequently found to be contaminated with Legionella pneumophila. ${ }^{5}$ He returned to the home that he shared with is mother in Porto (approximately $300 \mathrm{~km}$ from Vila Franca de Xira) on October 11 and again on the evening of October 19 (Fig. 1). His symptoms began on October 14, and on October 19, he had severe respiratory symptoms, including an intense cough. During that night, his mother (Patient 2) took care of him until he was admitted to the hospital (Centro Hospitalar do Porto) approximately 8 hours later. On October 22, he was transferred to another hospital to receive extracorporeal membrane oxygenation.

On October 27, Patient 2, who was a previously healthy 74-year-old woman, began to report fever, cough, and loss of appetite. She was admitted to the same hospital on November 3 with septic shock due to pneumonia, and she died on December 1. Patient 1 died on January 7, 2015.

Urine specimens obtained from both patients showed positive results on testing for legionella antigens with the use of a commercial enzyme immunoassay (Binax), and legionella was grown in culture from respiratory secretions. L. pneumophila serogroup 1 was identified, and strains were sent to the National Institute of Health in Lisbon for sequence-based typing and wholegenome sequencing (details are provided in the Methods section in the Supplementary Appendix, available with the full text of this letter at NEJM.org). Samples from the patients were obtained and processed more than 2 weeks apart (for culture and sequence-based typing) and 4 weeks apart (for whole-genome sequencing) to minimize the risk of cross-contamination. Both strains showed the novel ST1905 profile (identified as the causative strain in the cluster ${ }^{5}$ ), and whole-genome sequencing revealed no nucleotide differences within the region that spanned approximately $3.47 \mathrm{Mb}$ of the genome sequence. This genome matched the one identified in the cluster-related isolates.

An investigation headed by the General Directorate of Health showed that L. pneumophila ST1905-associated disease occurred in people who lived or stayed in Vila Franca de Xira between October 14, when the cluster began, and November 21, when it was considered to be controlled. Patient 2 had remained in Porto during these months, and to the best of our knowledge, she was the only person infected with L. pneumophila ST1905 who was not geographically linked to the cluster epicenter. Later investigation revealed that Patient 2 had never been to Vila Franca de Xira, and during the cluster period,

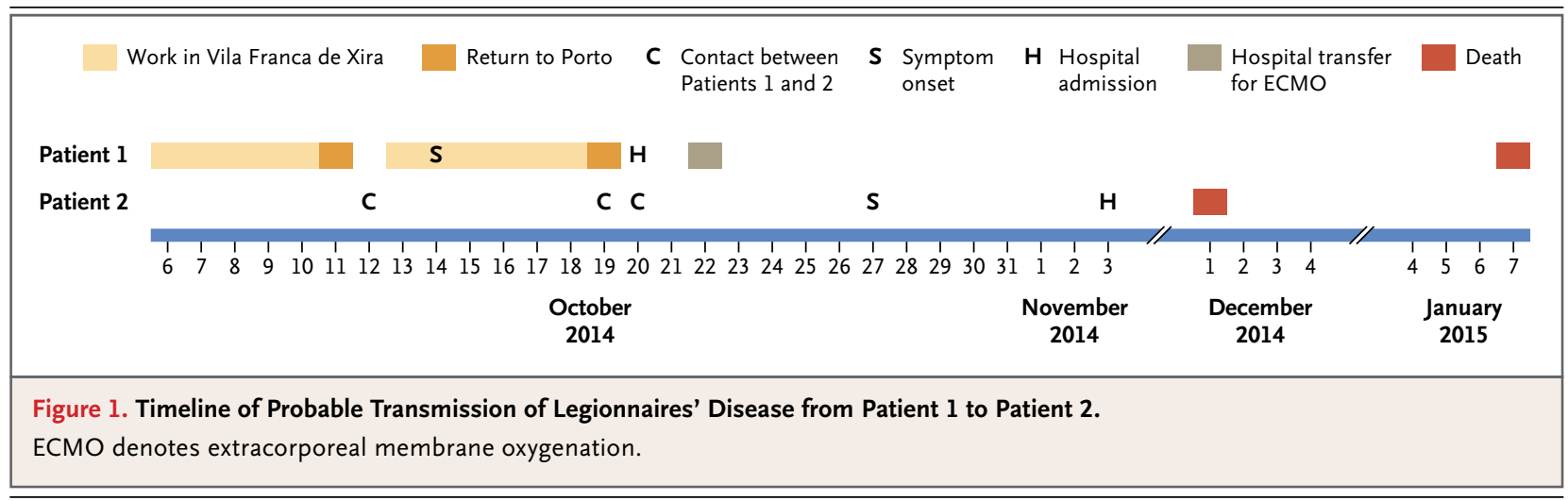


no additional cases of Legionnaires' disease occurred in Porto.

Patients 1 and 2 lived alone in Porto, and their house consisted of small nonventilated rooms without air-conditioning units or room humidifiers. Collected water samples from the bathroom and the kitchen and a swab of the shower drain were negative for legionella. Patient 1 did not take water from Vila Franca de Xira to Porto.

We suspect that person-to-person transmission probably occurred when Patient 2 cared for her severely ill son. Factors that suggest personto-person transmission are the severity of the respiratory symptoms in Patient 1 , the very close contact that occurred during the 8 consecutive hours when Patient 2 took care of Patient 1, and the small area of the nonventilated room where this contact took place. In addition, the timeline of the events was highly coherent (i.e., the symptoms in Patient 2 developed 1 week after the close contact with Patient 1; this is consistent with the typical incubation period of Legionnaires' disease - a median of 6 to 7 days). ${ }^{1}$

Ana M. Correia, M.D.

Northern Regional Health Administration

Porto, Portugal

acorreia@arsnorte.min-saude.pt

Jorge Gonçalves, M.D.

Porto Ocidental Health Centers Group

Porto, Portugal

João P. Gomes, Ph.D.

National Institute of Health

Lisbon, Portugal

\section{and Others}

A complete list of authors is available with the full text of this letter at NEJM.org.

Disclosure forms provided by the authors are available with the full text of this article at NEJM.org.

1. Phin N, Parry-Ford F, Harrison T, et al. Epidemiology and clinical management of Legionnaires' disease. Lancet Infect Dis 2014;14:1011-21.

2. Fields BS, Benson RF, Besser RE. Legionella and Legionnaires' disease: 25 years of investigation. Clin Microbiol Rev 2002; 15:506-26.

3. European Centre for Disease Prevention and Control. Legionnaires' disease in Europe, 2012. Stockholm: ECDC, 2014 (http:// www.ecdc.europa.eu/en/publications/Publications/legionnaires -disease-surveillance-2012.pdf).

4. Legionellosis - United States, 2000-2009. MMWR Morb Mortal Wkly Rep 2011;60:1083-6.

5. Shivaji T, Sousa Pinto C, San-Bento A, et al. A large community outbreak of Legionnaires disease in Vila Franca de Xira, Portugal, October to November 2014. Euro Surveill 2014;19: 20991.

DOI: 10.1056/NEJMc1505356

Correspondence Copyright @ 2016 Massachusetts Medical Society.

\section{NOTICES}

Notices submitted for publication should contain a mailing address and telephone number of a contact person or department. We regret that we are unable to publish all notices received. Notices also appear on the Journal's website (NEJM.org/medical-conference). The listings can be viewed in their entirety or filtered by specialty, location, or month.

\section{MAYO CLINIC SCOTTSDALE}

The following courses will be offered in Scottsdale, AZ, unless otherwise indicated: "Mayo Clinic Symposium on Anesthesia and Perioperative Medicine" (Feb. 10-13); "Enhancing Recovery After Surgery, 2016: Improving the Quality of Recovery for the Patient" (Phoenix, AZ, Feb. 12 and 13); "Mayo Clinic Gastroenterology and Hepatology 2016" (Feb. 25-28); "12th Annual Mayo Clinic Women's Health Update" (March 3-5); "27th Annual Family Medicine and Internal Medicine Update" (March 16-19); "Tackling Problematic Sinusitis: Module-Based Solutions" (Phoenix, AZ, March 31-April 2); "3nd Annual Southwest Laryngology Conference: Focus on Voice Disorders" (April 8-10); and "19th Annual Mayo Clinic Internal Medicine Update: Sedona 2016" (Sedona, AZ, Oct. 6-9, Oct. 20-23).

Contact Mayo School of Continuous Professional Development, Mayo Clinic, 13400 E. Shea Blvd., Scottsdale, AZ 85259; or call (480) 301-4580; or fax (480) 301-8323; or e-mail mca.cme@ mayo.edu; or see http://www.mayo.edu/cme.

\section{MAYO CLINIC}

The following courses will be offered in Rochester, MN, unless otherwise indicated: "Mayo Clinic Wound Symposium" (Feb. 18-20); "Commercial Driver Medical Examiner Training" (Jacksonville, FL, March 12; Pocono Manor, PA, June 18; Oct. 29); "Pain Medicine for the Non-Pain Specialist" (March 16-18); "SKIN: Practical Dermatology for the Generalist" (Chicago, April 15 and 16); "2016 Mayo Clinic T. Denny Sanford Pediatric Symposium" (April 22); "Comprehensive Management of Salivary Gland Disease" (May 12-14); "Mayo Clinic Physical Medicine \& Rehabilitation Board Review" (May 18-20); "Disorders of the Wrist" (May 19-22); and "5th Annual Comprehensive Sports Medicine Update and Board Review" (Minneapolis, June 22-25).

Contact the Mayo School of Continuous Professional Development, 200 First St. SW, Rochester, MN 55905; or call (800) 323-2688 or (507) 284-2509; or fax (507) 284-0532; or see https://ce.mayo.edu; or e-mail cme@mayo.edu.

\section{INTERNATIONAL SYMPOSIUM ON INTENSIVE CARE AND EMERGENCY MEDICINE (ISICEM)}

The symposium will be held in Brussels, March 15-18.

Contact Dominique Szyke, Erasme Hospital, Intensive Care Department, Route de Lennik 808, B-1070 Brussels, Belgium; or call (32) 255536 94; or fax (32) 2555 4555; or e-mail d.szyke@ intensive.org; or see http://www.intensive.org.

\section{ST WORLD CONGRESS ON HEART DISEASE}

The congress will be held in Boston, July 30-Aug. 1. Deadline for submission of abstracts is Feb. 25.

Contact Dr. Ascher Kimchi, Congress Chairman, International Academy of Cardiology, P.O. Box 17659, Beverly Hills, CA 90209; or call (310) 657 8777; or fax (310) 659 4781; or e-mail klimedco@ucla.edu; or see http://www.cardiologyonline.com.

THE JOURNAL'S WEB AND E-MAIL ADDRESSES

To submit a letter to the Editor: authors.NEJM.org For information about the status of a submitted manuscript: authors.NEJM.org

To submit a meeting notice: meetingnotices@NEJM.org The Journal's web pages: NEJM.org 